ing and memory remains to be established, the strengthening of support for a leading candidate cellular substrate is an encouraging observation that can be followed up in several ways. Firstly, it should be possible to examine the same cell population at the molecular and cellular levels in order to elucidate the processes underlying these modifications. Secondly, the changes in spatiotemporal patterns hint at restructuring of $\mathrm{KC}$ odor representations that might reflect a correlate of information storage. It may now be feasible, using similar imaging approaches but at single cell resolution, to resolve the details of the changes. Finally, it should be possible, albeit technically challenging, to take advantage of the possibility of behavioral measurements in combination with imaging to try to directly link KC changes to memory storage itself.

\section{REFERENCES}

Faber, T., Joerges, J., and Menzel, R. (1999). Associative learning modifies neural representations of odors in the insect brain. Nat. Neurosci. 2, 74-78.

Faber, T., and Menzel, R. (2001). Visualizing mushroom body response to a conditioned odor in honeybees. Naturwissenschaften 88, 472-476.

Galizia, C. G., and Menzel, R. (2000). Odour perception in honeybees: coding information in glomerular patterns. Curr. Opin. Neurobiol. 10, 504-510.
Hammer, M. (1997). The neural basis of associative reward learning in honeybees. Trends Neurosci. 20, 245-252.

Stopfer, M., and Laurent, G. (1999). Short-term memory in olfactory network dynamics. Nature 402, 664-668.

Szyszka,P., Galkin,A., and Menzel, R. (2008).Associative and non-associative plasticity in Kenyon cells of the honeybee mushroom body. Front. Syst. Neurosci. 2, 3 .

Received: 20 October 2008; published: 15 December 2008

Citation: Front. Neurosci. (2008) 2, 2: 131-132. doi: 10.3389/neuro.01.041.2008

Copyright: (c) 2008 Vicente and Mainen. This is an openaccess publication subject to an exclusive license agreement between the authors and the Frontiers Research Foundation, which permits unrestricted use, distribution, and reproduction in any medium, provided the original authors and source are credited.

\title{
Brain rhythms in the human medial temporal lobe
}

\section{Hans-Jochen Heinze*}

Department of Neurology, Otto-von-Guericke-University, Magdeburg, Germany

* Correspondence: hans-jochen.heinze@med.ovgu.de

\section{A commentary on}

Independent delta/theta rhythms in the human hippocampus and entorhinal cortex

by Florian Mormann, Hannes Osterhage, Ralph G. Andrzejak, Bernd Weber, Guillén Fernández, Juergen Fell, Christian E. Elger and Klaus Lehnertz

Ever since the discovery of the electroencephalogram (EEG), brain oscillations have been a major focus of neuroscientific research. The perpetual interactions among multiple network oscillators enable the brain to perform global computations on multiple spatial and temporal scales. A prominent example of interacting oscillations is the phenomenon of phase precession of place cells in the rodent hippocampus (O'Keefe and Recce, 1993). When the rat traverses a particular region in its environment, these cells fire periodic bursts at a slightly faster rhythm than the ongoing theta oscillation in the hippocampus. The firing thus occurs at increasingly early phase angles of the local field oscillations, and the spatial position of the animal can be decoded from these phase angles.

In this issue, Mormann et al. (2008) investigate oscillatory activity in the human hippocampus and in its main input structure, the entorhinal cortex. Using a unique data set of intracranial EEG signals directly recorded from the healthy medial temporal lobes of epilepsy patients with strictly unilateral seizure onset, the authors perform an elegant data analysis and provide evidence for the existence of independent theta generators in the human hippocampus and entorhinal cortex.

Of these two independent theta rhythms, the entorhinal rhythm is seen as being mediated by sensory and other cortical inputs, whereas the hippocampal rhythm is assumed to reflect theta activity autonomously generated within the hippocampus itself, mediated by inputs from the medial septum. Furthermore, the authors consider the hypothesis that the two independent rhythms represent the two oscillators of slightly different frequencies postulated by the interference model of theta phase precession (O'Keefe and Recce, 1993). Recent advances in recording technology that allow simultaneous recording of local field potentials and single-neuron activity in humans make this hypothesis an exciting and, more importantly, a testable one.

Previous studies on intracranial EEG recordings have shown that successful memorization of presented words is associated with an increase in entorhinalhippocampal theta coherence (Fell et al.,
2003). Mormann et al. now conjecture that the independent theta rhythms found in the entorhinal cortex and hippocampus may need to be actively synchronized to facilitate the synaptic plasticity involved in memory encoding. This hypothesis is backed by findings that the theta phase at which an action potential arrives in the rodent hippocampus determines the direction of plasticity, i.e. whether it results in long-term potentiation or depression. Synchronization of theta activity between the hippocampus and entorhinal cortex could thus create a slowly modulated facilitating state that provides a temporal basis for encoding or retrieval of separate items. A potential mechanism to generate the synchronization was discovered by Mormann et al. (2005). In a continuous word recognition paradigm, the authors found that the presentation of a visual stimulus causes a simultaneous phase reset of ongoing oscillatory activity in the hippocampus and entorhinal cortex.

Furthermore, the existence of independent theta rhythms plays an important role for a number of mechanisms related to gamma oscillations (Fries et al., 2007), as gamma activity has been found to be coupled to the phase of ongoing theta oscillations (Canolty et al., 2006; Demiralp et al., 2007; Mormann et al., 2005). 


\section{REFERENCES}

Canolty, R. T., Edwards, E., Dalal, S. S., Soltani, M., Nagarajan, S. S., Kirsch, H. E., Berger, M. S., Barbaro, N. M., and Knight, R. T. (2006). High gamma power is phase-locked to theta oscillations in human neocortex. Science 313, 1626-1628.

Demiralp, T., Bayraktaroglu, Z., Lenz, D., Junge, S., Busch, N.A., Maess, B., Ergen, M., and Herrmann, C. S. (2007). Gamma amplitudes are coupled to theta phase in human EEG during visual perception. Int. J. Psychophysiol. 64, 24-30.

Fell, J., Klaver, P., Elfadil, H., Schaller, C., Elger, C. E., and Fernández, G. (2003). Rhinal-hippocampal theta coherence during declarative memory formation: interaction with gamma synchronization? Eur. J. Neurosci. 17, 1082-1088.

Fries, P., Nikolić, D., and Singer, W. (2007). The gamma cycle. Trends Neurosci. 30, 309-316.

Mormann, F., Fell, J., Axmacher, N., Weber, B., Lehnertz, K., Elger, C. E., and Fernández, G. (2005). Phase/amplitude reset and theta-gamma interaction in the human medial temporal lobe during a continuous word recognition memory task. Hippocampus 15, 890-900.

Mormann, F., Osterhage H., Andrzejak R. G., Weber, B., Fernández, G., Fell, J., Elger, C. E., and Lehnertz, K. (2008). Independent delta/theta rhythms in the human hippocampus and entorhinal cortex. Front. Hum. Neurosci. 2, 3. doi: 10.3389/neuro.09.003.2008.
O’Keefe, J., and Recce, M. L. (1993). Phase relationship between hippocampal place units and the EEG theta rhythm. Hippocampus 3, 317-330.

Received: 05 November 2008; published: 15 December 2008

Citation: Front. Neurosci. (2008) 2, 2: 132-133. doi: 10.3389/neuro.01.045.2008

Copyright: (c) 2008 Heinze. This is an open-access publication subject to an exclusive license agreement between the authors and the Frontiers Research Foundation, which permits unrestricted use, distribution, and reproduction in any medium, provided the original authors and source are credited.

\section{Looking inward: the mind's eye focuses on mental representations}

\section{George R. Mangun* \\ Center for Mind and Brain, and Departments of Psychology and Neurology, University of California, Davis, USA \\ *Correspondence: mangun@ucdavis.edu}

\section{A commentary on}

Spatial attention can bias search in visual short-term memory

by Anna C. Nobre, Ivan C. Griffin and Anling Rao

In his visionary writings in the 19th century, American psychologist James (1890) wrote about attention describing it in part as:

"...the taking possession of the mind, in clear and vivid form, one out of what seem several simultaneously possible objects or trains of thought."

Over the course of the ensuing century, especially in the past 60 years, researchers investigating the mechanisms of attention have identified important behavioral and neural correlates of attention, which include the findings that attention influences the processing of sensory stimuli by improving perception and performance for attended stimuli versus unattended stimuli (e.g., Cherry, 1953; Posner, 1978), and that such effects can involve changes in sensory-neural signals early in the sensory hierarchy for auditory (e.g., Hillyard et al., 1973), visual (e.g., Van Voorhis and Hillyard, 1977) and somatosensory (e.g., Desmedt and Robertson, 1977) stimuli.

However, as James' quote makes it clear, selective attention not only involves select- ing between competing external signals, but also acting to select between internal and external signals, and perhaps as well between competing internal signals held in short- or long-term memory stores. I recall vividly that my late father George H. Mangun, a biochemist, could withdraw almost completely from the welter of our living room when my brother, sister and I were engaged in childhood mischief and mayhem. When he was focused on a difficult scientific problem, we had to physically leap on him to capture his attention. I asked him about this once, in amazement of his formidable mental sound-proofing, and he told me that he perfected the skill in college in order to study without being distracted. As a child I was not wholly convinced, but as an attention researcher (and a parent of two young boys myself!), I now understand the powerful nature of the human attention system for modulating sensory processing. Surprisingly, in contrast to work on the effects of attention on sensory inputs, very little work has addressed how attention can be turned inward to select from purely mental representations. There is no doubt that this paucity of research has to do with the simple fact that it is challenging to develop reliable measures of mental representations in the first place, and still harder to measure how such representations may be affected by other cognitive factors such as momentary attention.

Nobre et al. (2008), building on their and others' earlier work, have conducted a very interesting study that does just this; they have investigated, using behavioral and physiological measures, the effects of attention on representations held in one form of mental store - visual short term memory (VSTM). VSTM is a short-term (a few seconds) limited-capacity store of visual information (e.g., Zhang and Luck, 2008). Nobre et al. (2008) investigated whether the retrieval of information from VSTM could be influenced by focused attention. The authors utilized a paradigm similar to those used in studies of the effects of covert selective attention on vision using predictive precues (e.g., Mangun and Hillyard, 1991). However, instead of precuing the location to which attention should be directed in future, the authors used retrodictive cues (spatial retro-cues) that indicated the location of a relevant target in an array presented in the past (1-2 s previously) with $100 \%$ probability. In comparison to neutral retro-cues that gave no information about the likely location in the array of the relevant item, performance to indicate whether a subsequently presented probe stimulus had in fact been anywhere in the array was improved with the spatial retro-cues. Importantly, the 